\title{
Codistances in buildings
}

\author{
Bernhard Mühlherr Hendrik Van Maldeghem
}

\begin{abstract}
A codistance in a building is a twinning of this building with one chamber. We study this local situation and prove that affine Bruhat-Tits buildings defined over $p$-adic numbers do not admit a codistance.
\end{abstract}

Keywords : twin building, $p$-adic building, affine building

MSC 2000: 51E24

\section{Introduction}

Twin buildings have been introduced by Ronan and Tits (see [13]) to give a geometric interpretation of the Kac-Moody groups. Roughly, a twin building is a pair of buildings of the same type endowed with a codistance between the chambers of the different buildings. The codistance takes values in the corresponding Coxeter group (the Weyl group of the buildings). The idea is that, given a chamber $c$ in one building, there is a set $c^{\mathrm{op}}$ of chambers in the other building opposite $c$, and the codistance to $c$ measures the distance to this set $c^{\mathrm{op}}$ "in a consistent way", i.e., according to some rules which are complementary to the rules of the Weyl distance. As always happens with important mathematical objects, people try to find axioms which characterize the objects, but which, at the same time, are easier to check, or ostensibly weaker. For twin buildings, the notion of a 2-twinning, introduced by the first author in [7], was important in this direction, but Abramenko and the second author showed in [2] that a 1-twinning (which is weaker than a 2-twinning) is not sufficient for a twinning (and they give a sufficient additional axiom). Now, 1- and 2-twinnings are still global conditions in that all chambers play the same role. Another, local way, to weaken the axioms is to fix one chamber and require the codistance axioms only with respect to that chamber; this approach yields precisely the axioms of a codistance on a given building. Up to now, it has been an open question whether 








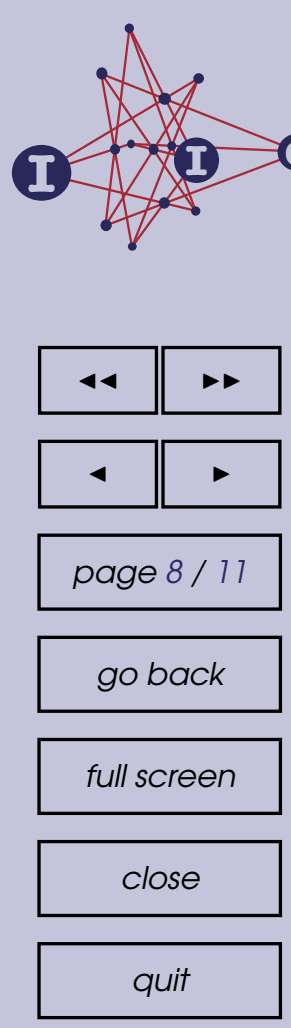

apartment containing both of them and if they are parallel in that apartment as hyperplanes. We refer to [4] for details.

Let $j \in S$ and let $R$ be a residue of cotype $j$ of an affine building $\mathbf{B}=(C, \delta)$ of type $(W, S)$. Let $\Sigma$ be an apartment of $\mathbf{B}$ intersecting $R$ nontrivially. Then we call $R$ special if for every wall $W$ in $\Sigma$ there is a parallel wall $W^{\prime}$ containing a panel both of whose chambers belong to $R$. This definition is independent of $\Sigma$. The wall $W^{\prime}$ above is said to cut $R$ in halves.

Let $R$ be a special residue in $\mathbf{B}$, and let $\Sigma$ be an apartment meeting $R$ nontrivially (by which we mean that $\Sigma$ and $R$ have chambers in common). Let $c \in C$ belong to $R$ and $\Sigma$. The intersection of all roots in $\Sigma$ whose bounding walls cut $R$ in halves and which contain $c$ is called a sector emerging from $R$. The sectors emerging from $R$ form the chamber set of a spherical building of the same type as $R$. Two chambers of this spherical building are adjacent if the corresponding sectors are contained in a common apartment and if they contain respective adjacent chambers of $\mathbf{B}$. This spherical building is called the building at infinity and denoted by $\mathbf{B}_{\infty}$. Its isomorphism type is independent of the special residue $R$ (and the canonical isomorphism between the buildings at infinity defined using $R$ and another special residue $R^{\prime}$ maps a sector to a sector in such a way that the intersection of these two sectors contains a sector itself). In particular, $\mathbf{B}_{\infty}$ can be represented with sectors emerging from any special residue. Also, the family of apartments of $\mathbf{B}$ is in bijective correspondence with the family of apartments of $\mathbf{B}_{\infty}$. We refer to [4] and [12].

Lemma 6.1. Let $\mathbf{B}=(C, \delta)$ be a building of affine type. Suppose there is a special thick residue $R$ such that $\mathbf{B}$ is a blow-up of $R$. Then the building $\mathbf{B}_{\infty}=\left(C_{\infty}, \delta_{\infty}\right)$ at infinity is isomorphic to $R$.

Proof. There is a natural epimorphism $\eta: \mathbf{B}_{\infty} \rightarrow R$ mapping a chamber $c_{\infty}$ of $\mathbf{B}_{\infty}$ emerging from $R$ to the unique chamber in $c_{\infty} \cap R$. Clearly, this mapping preserves adjacency of chambers, and hence maps non-opposite chambers onto non-opposite chambers. Now let $c_{\infty}^{1}$ and $c_{\infty}^{2}$ be two opposite chambers of $\mathbf{B}_{\infty}$, and let $\Sigma_{\infty}$ be an apartment of $\mathbf{B}$ containing $c_{\infty}^{1}$ and $c_{\infty}^{2}$. Then there is a unique apartment $\Sigma$ of $\mathbf{B}$ containing sectors $d_{\infty}^{1}$ and $d_{\infty}^{2}$ at bounded distance from $c_{\infty}^{1}$ and $c_{\infty}^{2}$, respectively. Since $\mathbf{B}$ is a blow-up of $R, \Sigma \cap R$ is nonempty and hence $c_{\infty}^{1}$ and $c_{\infty}^{2}$ are contained in $\Sigma$ (as all 'translates' in $\Sigma$ of $d_{\infty}^{1}$ and $d_{\infty}^{2}$ are at bounded distance from $d_{\infty}^{1}$ and $d_{\infty}^{2}$, respectively, and some translate emerges from $R$ ). Since $c_{\infty}^{1}$ and $c_{\infty}^{2}$ are opposite, also the chambers $R \cap c_{\infty}^{1}$ and $R \cap c_{\infty}^{2}$ are opposite (as the epimorphism mentioned above is bijective in the apartment $\Sigma$ ).

Hence $\eta$ preserves opposition and non-opposition. By Corollary 5.2 of [1], $\eta$ extends uniquely to an isomorphism. 


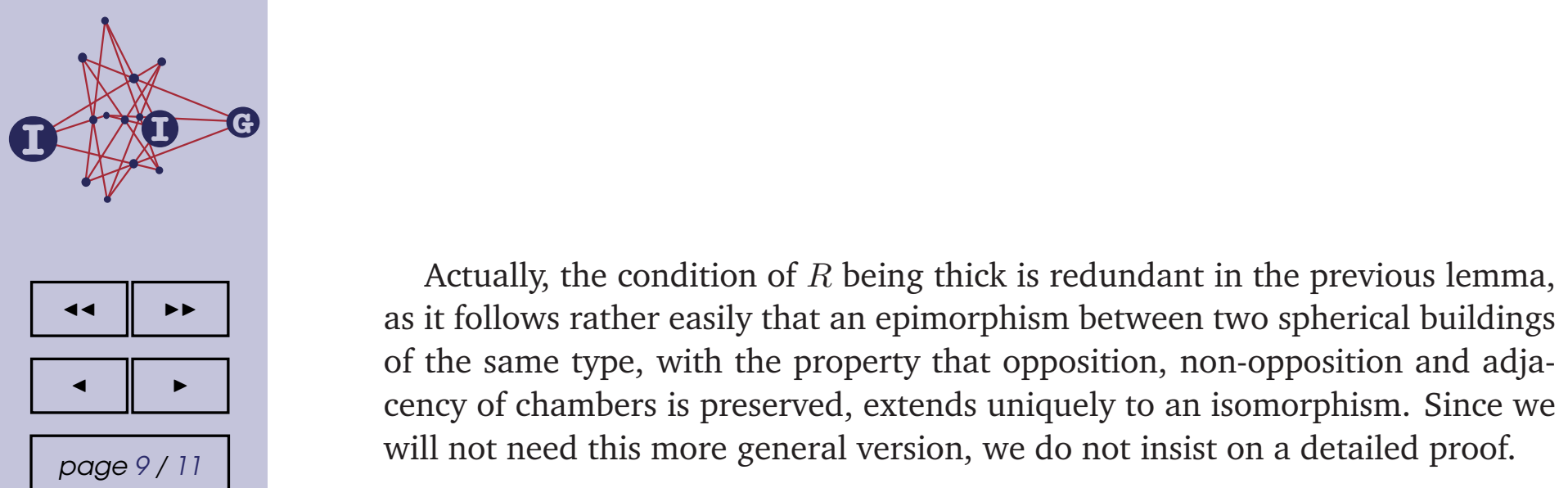

Lemma 6.2. Let $\mathbf{B}=(C, \delta)$ be an affine building, let $C^{\prime}$ be a firm convex subset of $C$ and put $\mathbf{B}^{\prime}:=\left(C^{\prime},\left.\delta\right|_{C^{\prime} \times C^{\prime}}\right)$. Then the building at infinity of $\mathbf{B}^{\prime}$ is a subbuilding of the building at infinity of $\mathbf{B}$.

full screen

close

Proof. This follows directly from the fact that every sector of $\mathbf{B}^{\prime}$ is a sector of $\mathbf{B}$.

Let $\mathbf{B}=(C, \delta)$ be an affine building and let $\delta^{*}: C \rightarrow W$ be a codistance. Choose a spherical residue $R$ of $\mathbf{B}$ containing a chamber in $\delta^{* \text { op }}$. By the previous sections, there is a convex sub-building $\mathbf{B}^{\prime}$ of $\mathbf{B}$ of the same type which is a blowup of $R$. The building at infinity of $\mathbf{B}^{\prime}$ is canonically isomorphic with $R$ and it is a subbuilding of the building at infinity of $\mathbf{B}$. So we have shown:

Theorem 6.3. Let $\mathbf{B}=(C, \delta)$ be a thick affine building, and let $\delta^{*}$ be a codistance on $\mathbf{B}$. Then every special residue containing a chamber of $\delta^{* \mathrm{op}}$ is a subbuilding of the building at infinity of $\mathbf{B}$ of the same type.

Our ultimate goal is to prove that an irreducible Bruhat-Tits building defined over the $p$-adic numbers, and of rank at least 3, does not admit a codistance. We will need the following fact about irreducible spherical Moufang buildings of rank at least 2. A proof for the rank 2 case is Lemma 5.2.2 of [14]. The arguments given there make implicit use of Tits' rigidity theorem [11, Theorem 4.1.1]. They generalize to the higher rank case without any problem.

Proposition 6.4. Let $\mathbf{B}=(C, \delta)$ be an irreducible spherical Moufang building of rank at least 2 and let $C^{\prime}$ be a thick convex subset of $C$. Then $\mathbf{B}^{\prime}:=\left(C^{\prime},\left.\delta\right|_{C^{\prime} \times C^{\prime}}\right)$ is a spherical Moufang building of the same type. Moreover, if $\alpha \subset C^{\prime}$ is a root of $\mathbf{B}^{\prime}$ and $U_{\alpha}^{\prime}$ is the corresponding root group with respect to $\mathbf{B}^{\prime}$, then $\alpha$ is also a root of $\mathbf{B}$ and $U_{\alpha}^{\prime}$ injects canonically into the corresponding root group with respect to $\mathbf{B}$.

The irreducible spherical Moufang buildings of rank at least 2 are classified and one easy consequence of this classification is the fact that one can associate a characteristic to each such building. Its characteristic is defined to be $0(p$, respectively) if the root groups are torsion free ( $p$-groups, respectively). The characteristic of the building at infinity $\mathbf{B}_{\infty}$ of a Bruhat-Tits building $\mathbf{B}$ coincides with the characteristic of the global field, whereas the characteristic of a 

